\title{
Pratiques
}

Linguistique, littérature, didactique

$159-160 \mid 2013$

Le figement en débat

\section{Le phénomène du détournement dans le langage des jeunes}

Silvia Palma

\section{(2) OpenEdition}

\section{Journals}

Édition électronique

URL : http://journals.openedition.org/pratiques/2853

DOI : $10.4000 /$ pratiques. 2853

ISSN : 2425-2042

Éditeur

Centre de recherche sur les médiations (CREM)

Édition imprimée

Date de publication : 15 décembre 2013

Pagination : 98-108

\section{Référence électronique}

Silvia Palma, «Le phénomène du détournement dans le langage des jeunes », Pratiques [En ligne], 159-160 | 2013, mis en ligne le 30 juin 2016, consulté le 30 avril 2019. URL : http:// journals.openedition.org/pratiques/2853; DOI : 10.4000/pratiques.2853

(c) Tous droits réservés 


\title{
Le phénomène du détournement dans le langage des jeunes
}

\author{
Silvia Palma
}

Université de Reims - CIRLEP EA 4299

\section{Introduction : un défigement devenu détournement}

Au départ, le titre de cet article faisait intervenir la notion de défigement, une notion que nous avions utilisée à plusieurs reprises pour expliquer les diverses transformations que peuvent subir notamment les proverbes et les locutions, à des fins humoristiques ou ironiques (cf. Palma 2007, 2008, 2009). Quelques exemples de ce procédé : Qui vole une puce vole un bus (sur la base de «Qui vole un œuf vole un bœuf») ; Qui trop embrase, mal éteint (sur la base de "Qui trop embrasse, mal étreint »); La Corse, tu l'aimes ou tu l'acquittes (détournement de «Tu l'aimes ou tu la quittes », à propos du procès d'Ivan Colonna, Le Canard Enchaîné, 7/11/2007).

Certains auteurs ayant travaillé sur la question du défigement linguistique ont préféré le terme de désautomatisation, notamment Zuluaga (1999, p. 541) qui pro-

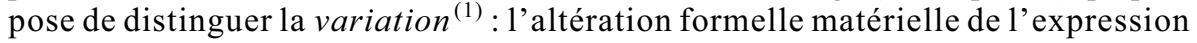
figée, et la désautomatisation ou défigement, qui renvoie aux effets de sens qui découlent de l'altération ${ }^{(2)}$.

L'existence de variations ne dément pas le figement, au contraire, la variation ne fait que confirmer celui-ci, elle s'appuie sur ce figement existant, à des fins expressives bien précises. Contrairement aux éléments figés de départ, les variations ne sont pas elles-mêmes figées : d'une part, elles constituent des créations dans l'acte de parole ou d'écriture au cours duquel elles apparaissent ; d'autre part, leur interprétation dépend directement du texte et du contexte d'apparition.

(1) Zuluaga utilise les termes «variación » et « desautomatización ».

(2) Le phénomène de défigement a été étudié également par B.-N. Grünig (1990), Schapira (1999), F. Rastier (1997), A. Gresillon et D. Maingueneau (1984), P. Barta (2005 et 2006). En dehors de ces études spécifiques, des remarques sur la possibilité de défigement apparaissent également dans les nombreuses études consacrées aux séquences figées de la langue. 
D'un point de vue diachronique, il est possible qu'une variation (donc une innovation) soit à l'origine d'un changement ou d'une variante, si elle arrive à se répandre et à être adoptée par une partie plus ou moins importante de la communauté linguistique. Elle deviendra, dans ce cas-là, une unité de la langue ${ }^{(3)}$.

En plongeant dans le corpus « langage des jeunes» - et sans doute prise dans la dynamique et la créativité de celui-ci —, nous avons tout d'abord envisagé de rapprocher les deux phénomènes. Or, il ne s'agit pas vraiment de défigement dans ce cas, puisque l'essentiel des transformations dans le langage des jeunes concerne le lexique plutôt que les éléments figés de la langue (encore faudrait-il se mettre d'accord sur les limites de cette notion). Nous avons donc finalement choisi la notion de détournement qui, tout en faisant allusion au jeu consistant à partir d'un élément connu pour en créer un autre, ne prend pas appui pas sur la notion d'élément figé.

\section{Langage des jeunes? Parlers jeunes? : une notion difficile à délimiter}

Dans la riche présentation de leur étude consacrée aux catégories « jeunes » et « parlers jeunes », M. Auzanneau et C. Julliard (2012) s'interrogent sur la nature et l'étendue de ces notions, en observant leur évolution dans les études sociolinguistiques. Dans cet objectif, les auteurs passent en revue divers travaux dont l'objectif était de déterminer si l'usage de certains traits était spécifique à la jeunesse, si cette période de la vie était une étape marquée culturellement et linguistiquement, et s'il existait des formes et/ou des registres spécifiques et donc non partagés des jeunes. Ces études ont permis de conclure à l'existence d'un lien entre l'usage de traits non standard et le fait, pour les adolescents, de participer à une culture vernaculaire de groupes de pairs.

Il est à noter que dans les nombreux travaux inspirés de l'approche de W. Labov, les chercheurs se sont focalisés sur les aspects identitaires des locuteurs, c'est-àdire que l'âge a été considéré en relation avec d'autres facteurs, notamment le niveau d'études, la mobilité géographique et sociale, le type de relations, etc. et non pas comme le seul critère déterminant ${ }^{(4)}$.

Bien entendu, les travaux passés en revue ne constituent pas un ensemble homogène : ils ont souvent été menés avec des objectifs différents, ils ont porté sur des réalités linguistiques spécifiques, et les approches choisies n'étaient pas forcément les mêmes. Pour ce qui est des études consacrées aux regroupements de jeunes en milieu urbain, elles sont devenues progressivement une partie incontournable de la sociolinguistique urbaine, sans doute du fait qu'elles ont permis, entre autres, d'enrichir et de mieux comprendre la variation dans les usages oraux de la langue, et d'affiner des questionnements relatifs à la pertinence sociale et linguistique de l'âge.

Il s'avère d'ailleurs fondamental d'insister sur le fait que ce type de catégorie

(3) Sur ce point en particulier, $c f$. Cusimano (2012).

(4) Dans le même sens, M. Auzanneau et C. Julliard (op. cit, p. 9) précisent que, « la jeunesse n'est donc pas, dans nos recherches, le point de vue central autour duquel s'organisent les constats portés sur l'identité des locuteurs, leurs réseaux relationnels ou leurs pratiques. Elle n'est qu'une des dimensions de cette identité et les locuteurs, par leurs pratiques in situ, peuvent ou non la rendre pertinente ». 
n'est pas à proprement parler une donnée mais une construction, comme l'avait notamment signalé P. Bourdieu. M. Auzanneau et C. Julliard (op. cit., p. 12) le rappellent dans les termes suivants :

Il apparaît que, souvent floue ou arbitraire et essentiellement envisagée d'un point de vue occidental, tant par les locuteurs que par les chercheurs, la catégorie jeune, tel que le rappelle Bourdieu (1984) n'est pas une donnée mais une construction. Elle est à préciser et à conceptualiser, à la fois d'un point de vue global - la culture, l'organisation sociale, les enjeux sociaux, le contexte socioéconomique, les conditions de vie, les pratiques sociales, etc. - et d'un point de vue local - la construction discursive des significations sociales telles que le positionnement interpersonnel, l'identification ou la différenciation, etc. Bien que les limites temporelles dévolues à la jeunesse soient variables, il est communément admis en linguistique, comme en sciences sociales ou en psychologie sociale (Bourdieu, 1984 ; Chambers 1998 ; Lamizet, 2004) que la jeunesse souvent associée à l'adolescence correspond à une période de transition entre l'enfance et l'âge adulte pendant laquelle le sujet social dépourvu de responsabilité et de pouvoir est mis « hors jeu » socialement (Bourdieu, 1984 ; p. 147 ; Chambers 1998 ; Lamizet, 2004).

Un dernier aspect à considérer est le fait que les catégories - en l'occurrence, la catégorie « jeune»- se construisent dans le cadre d'une interaction. Ainsi, un seul et même locuteur se comportera différemment en fonction des interlocuteurs participant à l'interaction. Dans le cas qui nous occupe, le jeune ne s'exprimera pas de la même façon lorsqu'il s'adresse à ses pairs et lorsqu'il est en relation avec des adultes, dans une situation plus ou moins formelle, pour ne prendre que deux cas bien différenciés. Notre étude concerne le premier cas de figure, car le choix de certains traits linguistiques permet au jeune de construire symboliquement des clôtures linguistiques, des facettes identitaires et des rapports de place de pair à pair $^{(5)}$.

\section{Caractéristiques générales du langage des jeunes et analyse de quelques stratégies récurrentes en français}

Divers traits ont été identifiés comme typiques du langage des jeunes ${ }^{(6)}:$ du point de vue phonétique, les aspects suprasegmentaux (intonation, débit « haché », déplacement de l'accentuation vers la pénultième syllabe...), la réalisation glottalisée de /r/, l'affrication des plosives vélaires et dentales en position prévocalique, la présence d'un e muet prépausal; du point de vue de la morphosyntaxe, les formes verbales non conjuguées, le changement de construction de certains verbes (assurer et craindre utilisés comme intransitifs, par exemple), le changement de catégorie grammaticale pour grave, trop, genre, pour ne citer que les cas les plus répandus.

D'autres aspects souvent mentionnés sont le vocabulaire limité et répétitif (uti-

(5) Pour des études de cas, $c f$. par exemple Laks (1983); Armstrong et Jamin (2002); I. Léglise et M. Leroy (2008), à propos du regard des médiateurs urbains sur le langage des jeunes; M. Auzanneau, M. Leclère-Messebel et C. Julliard (2012), sur les jeux de rôles dans le cadre d'un atelier de formation de la protection Judiciaire de la Jeunesse.

(6) Pour la caractérisation des différents aspects, cf. par exemple Gadet (2007), 120-131, M. Abecassis, L. Ayosso et E. Vialleton (2007) ; Alain Rey et Disiz La Peste (introduction au Lexik des cités, 2007). 
lisation abusive de faire, par ex : ça le fait, ça le fait pas, ça le fait trop...), les emprunts, notamment à l'arabe, la profusion d'expressions grossières, la troncation par apocope (biz pour business) ou par aphérèse (blème pour problème, leur pour contrôleur), la réduplication (leurleur pour contrôleur, zonzon pour prison), l'utilisation de formules du type de « $\mathrm{N}$ de chez $\mathrm{N} »$ pour indiquer une valeur superlative (Ce mec est nul de chez nul).

S'agissant d'un langage éminemment oral, certains phénomènes prennent une importance particulière, par exemple la façon de s'adresser à ses pairs, point sur lequel nous reviendrons plus loin, en rapport avec les insultes et les vannes.

\section{La création lexicale ${ }^{(7)}$}

Avant d'observer de plus près quelques procédés très fréquents de création lexicale dans cette variante linguistique, précisons que notre étude ne se propose pas de faire une présentation exhaustive des procédés, mais simplement d'illustrer, à travers quelques cas, la notion de détournement en jeu.

\section{Le "verlan enrichi"}

Le procédé d'inverser les syllabes d'un mot n'est certes pas nouveau. Ce qui a attiré notre attention c'est la poursuite des transformations, une fois le mot «verlanisé », soit à travers des modifications graphiques, soit par le biais d'ajouts, d'apocopes, etc. Au point que le résultat final ne permet pas toujours de reconnaître aisément le mot français à l'origine de ces transformations.

Ce procédé affecte notamment les adjectifs et les verbes, et la transformation s'accompagne souvent d'un changement ou d'une restriction du sens, comme on peut le constater dans les adjectifs suivants :

Auch : de chaud. Deux sens possibles pour 1'adjectif transformé : 1) difficile, compliqué ( $C a$ va être auch pour sortir ce soir, mon père veut pas). Synonyme : ghetto ; 2) dangereux (Le parking de la gare, c'est auch). Synonyme: machope.

Chanmé : de méchant. Sens : génial, formidable. (Le cours, il était chanmé, on a trop bien rigolé avec le prof). Synonymes : bad, hard.

Chelou : de louche. Deux sens possibles : 1) bizarre (T'es chelou, toi! Qu'estce qui te prend de t'habiller comme ça?) ; 2) douteux (Cette viande, elle est chelou, elle a une drôle d'odeur).

Kéblo : de bloqué. Deux sens possibles : 1) être retenu quelque part (donc plutôt valeur de participe passé) ; 2) inhibé, maladroit. Le nom kéblo, pour sa part, correspond plutôt au sens d'idiot (Ce mec-là, c'est un kéblo, il a vendu sa télé pour s'acheter un magnétoscope). Synonyme : P4.

Dans le cas des verbes, on constate un glissement sémantique similaire :

Pécho : de choper. Deux sens possibles : 1) attraper. Synonyme : serrer ; 2) conclure [initier une relation] avec quelqu'un. (J'ai pécho le mec dont je t'ai parlé à la soirée d'hier, je suis trop contente).

(7) La plupart des exemples cités proviennent de l'ouvrage Lexik des cités illustré (2007), préface d'Alain Rey et Disiz La Peste. Nous avons volontairement laissé de côté les emprunts à d'autres langues. 
Pouille-D : de dépouiller. Deux sens possibles : 1) dévaliser ; 2) voler (Je suis dégoûté, je me suis fait pouille-d mon vélo).

Veski : esquiver. Trois sens possibles, tous relativement proches : 1) éviter une personne (Son père la cherche partout, mais elle le véski) ; 2) éluder un sujet (Arrête de véski quand je te parle de choses sérieuses) ; 3 ) fuir une situation ( $J$ 'ai vu le pit-bull foncer sur moi, j'ai véski direct).

\section{La création par suffixation}

Le procédé de suffixation, extrêmement fréquent dans les langues, présente certaines particularités dans le langage des jeunes, notamment un découpage en morphèmes qui ne répond que très rarement aux règles habituelles. Ainsi, par exemple, la transformation des noms camion et pavillon en kamtar et pavtar supposerait l'existence d'une base cam-devenue kam et d'une autre pav-auxquelles viendrait s'ajouter le suffixe -tar.

D'autres cas de création se rapprochent davantage des procédés habituels. Ainsi, le suffixe -ard, présent par exemple dans campagnard, montagnard, routard et les plus familiers taulard, zonard, avec le sens de " personne qui vit à la campagne, à la montagne, etc. » permet de créer des noms comme blédard (e) pour désigner quelqu'un qui vient du bled ${ }^{(8)}$.

\section{Le glissement de sens sans modification de la forme du mot}

Il est également fréquent de trouver dans ce corpus des mots de la langue courante qui n'ayant pas subi de transformation morphologique, acquièrent tout de même des nuances différentes. En s'appuyant à la fois sur le sens du mot d'origine et sur le contexte, ces nouvelles valeurs peuvent être déduites plus ou moins facilement, selon les cas.

Cash : le sens d'origine " payer en espèces » ${ }^{(9)}$ a donné lieu à la valeur de $d i^{-}$ rect, franc lorsque cash est utilisé comme adjectif (Ce mec, il est trop cash) ou directement, franchement lorsque le mot est utilisé comme adverbe (Ils se sont expliqué cash).

Se capter : sans doute en rapport avec le sens de « réussir à établir une communication », se capter peut prendre le sens de se rejoindre ou de rester en contact, selon les contextes.

Comme indiqué dans le point précédent, le changement sémantique correspond souvent à une spécialisation, à une restriction du sens d'origine :

Bail : le sens contractuel de bail $^{(10)}$ s'est considérablement spécialisé. Ainsi, bail renvoie dans le langage des jeunes à «plan amoureux » (J'ai enfin un

(8) Jadis employé dans le jargon militaire pour désigner un paysan ou un soldat, blédard vient de l'arabe dialectal maghrébin blad qui vient lui-même de bilad en arabe classique, signifiant « contrée », « terrain», « pays ». A noter qu'en plus de ce sens, blédard signifie également « ringard», « décalé ». (Lexik des cités, p. 69)

(9) Emprunté au français caisse, issu du provençal caissa, lui-même tiré du latin capsa, le mot cash en anglais est attesté depuis le XVI ${ }^{\mathrm{e}}$ siècle au sens d'argent, monnaie, sens lié au contenu d'une «boîte, coffre, caisse de marchand». La similitude phonétique avec le mot kasu en tamil, qui désigne dans le sud de l'Inde une «pièce de monnaie courante », aurait fini par établir solidement la référence à l'argent sonnant et trébuchant, par opposition au crédit. » (Lexik des cités, p. 95).

(10) Contrat par lequel une des parties (le bailleur) s'oblige à faire jouir l'autre (le locataire) 
bail, ça fait plaisir), mais aussi à « un commerce informel, un arrangement » (Toi et moi, faut qu'on trouve un bail à faire pour cette histoire de voitures) et par extension, aux nouvelles (C'est quoi les bails? au sens de Quoi de neuf?).

Dossier : le sens administratif de dossier ${ }^{(11)}$ est devenu clairement négatif, car le mot renvoie dans le corpus considéré à « des informations pouvant ridiculiser ou compromettre quelqu'un ».

D'autres glissements de sens renvoient au champ lexical du sport. Ainsi, certains verbes désignant des actions précises dans le cadre d'une activité sportive ont pris dans le langage des jeunes un sens différent, bien qu'en rapport plus ou moins étroit avec le premier : le verbe dribbler, désignant en sport l'action de courir en poussant devant soi le ballon sans en perdre le contrôle, a vu son sens se restreindre à «tromper son amoureux ». D'une manière similaire, tacler, désignant l'action de faire obstruction à l'adversaire dans le but de lui prendre la balle, a vu son sens se restreindre au fait de "devancer quelqu'un dans une rivalité amoureuse » (Ça fait une semaine que je drague la standardiste, je me suis fait tacler par un collègue, il sort avec elle demain).

\section{Le mode d'adresse}

Un autre aspect permettant d'illustrer le phénomène de détournement est l'utilisation de la part des jeunes — extrêmement abondante, d'ailleurs — d'insultes ou de termes connotés négativement pour s'adresser aux proches, aux membres du groupe. Ainsi, par exemple, sont cités comme synonymes de frère dans Lexik des cités des termes à connotation aussi diverse que : gros, ma came, ma couille, ma gueule, négro, poto, kho (emprunt)...

La présence de ces termes, dont certains sont connotés très négativement dans d'autres contextes, est à rapprocher de la notion d'insulte de solidarité, ou de celle de vanne entre pairs. La question de l'usage des insultes chez les jeunes a été étudiée par divers auteurs ${ }^{(12)}$, prenant comme base les études sur les injures de D. Lagorgette ${ }^{(13)}$. Ainsi, D. Caubet, qui s'est penché sur le rôle des insultes, injures et vannes en France et au Maghreb signale :

Il semble que les vannes aient deux caractéristiques essentielles qui les distinguent des injures, outre le fait que l'activité semble réservée aux plus jeunes : elles s'adressent à des pairs, à des membres du groupe ; le contenu des vannes est aberrant et ne se rapproche pas de la vérité. Cependant, il existe des cas où l'on touche à des situations ayant existé et la frontière est ténue. (D. Caubet $(2008,113)$ ).

D. Lepoutre, pour sa part, classe explicitement les vannes parmi les insultes rituelles :

d'une chose pendant un certain temps moyennant un certain prix (loyer) que celle-ci s'oblige à payer (Le Nouveau Petit Robert, édition 1994)

(11) Ensemble de pièces relatives à une affaire et placées dans une chemise. La pochette, la chemise qui contient ces pièces. L'ensemble des renseignements contenus dans ces pièces. ( $L e$ Nouveau Petit Robert, édition 1994).

(12) Cf. par exemple D. Lepoutre (1997), D. Caubet (2008), I. Léglise et M. Leroy (2008).

(13) Pour les travaux de D. Lagorgette, nous renvoyons notamment à Lagorgette (2009). Pour les études sur les insultes en espagnol, $c f$. notamment Luque JdD., A. Pamiés ,et J. Manjón (1998) et (2000). 
Le terme vanne - d'usage populaire plus ou moins argotique — désigne communément toutes sortes de remarques virulentes, de plaisanteries désobligeantes et de moqueries échangées sur le ton de l'humour entre personnes qui se connaissent ou du moins font preuve d'une certaine complicité. Le principe des vannes repose fondamentalement sur la distance symbolique qui permet aux interlocuteurs de se rallier ou même de s'insulter mutuellement sans conséquences négatives. (D. Lepoutre $(1997,137)$ [cité par Caubet]).

Ces insultes rituelles serviraient donc de marque de solidarité, d'intimité, et non pas d'agression, tant que les locuteurs appartiennent au même groupe. Au-delà, elles gardent toute leur force négative. Ces insultes peuvent concerner directement les interlocuteurs, mais aussi des personnes qui leur sont proches, leurs familles (avec une nette préférence pour la sœur ou la mère de l'interlocuteur) :

Cette pratique adolescente des insultes rituelles se donne à voir sur son aspect le plus original et le plus frappant dans un type particulier de vanne - type modèle en quelque sorte - qui n'existe, semble-t-il, que dans ce contexte social. Ce sont les vannes sur la mère, ou vannes référencées, qui visent indirectement, c'est-àdire par parents interposés, les personnes « insultées ». (D. Lepoutre $(1997,138)$ [cité par Caubet]).

Pour D. Caubet, une différence importante est à signaler sur ce point entre la France et le Maghreb :

Les jeunes distinguent clairement les vannes référencées des vannes directes; et c'est sans doute là que se situe la différence fondamentale avec le Maghreb, où on ne peut pas plaisanter sur les parents sans que cela soit considéré comme un manque de respect. (D. Caubet $(2008,120))$.

Il est à noter également que la valeur des termes évolue très rapidement, comme l'ont constaté par exemple, I. Léglise et M. Leroy (2008), dans le cadre de leur étude sur le rôle des médiateurs urbains dans les cités. En effet, quelques-uns des termes qui étaient pour les médiateurs extrêmement forts à l'époque de leur adolescence, sont devenus en quelques années de simples éléments phatiques :

Ainsi, les termes de fils de pute ou de bâtard, considérés par les médiateurs [des personnes entre 20 et 30 ans] comme des insultes suprêmes, en même temps qu'emblématiques, et très clairement catégorisés comme " mots qui font mal ", sont de fait passés du côté des mots affectueux chez les adolescents actuels. [...] Par ailleurs, on constate également le figement des termes bâtard et fils de pute à la forme masculine, même parmi les filles, ce qui semble appuyer l'hypothèse d'une utilisation en tant que phatiques. (I. Léglise et M. Leroy (2008, 164-165)).

Afin de déterminer si les caractéristiques étudiées jusque là se vérifient dans d'autres langues que le français, nous consacrerons la dernière partie de cette étude à la présentation des principaux traits du parler jeune en espagnol ${ }^{(14)}$.

(14) Comme il a été indiqué par divers auteurs, la variété de parlers jeunes est immense et ce qui est valable pour les jeunes d'une ville ou d'une région ne le sera pas forcément pour ceux d'une autre ville, une autre région. Les exemples proposés ont donc toujours, forcément, une validité restreinte. Nous nous sommes bornée ici à des exemples correspondant à la variante péninsulaire, mais il serait intéressant d'élargir l'étude aux variantes jeunes de l'espagnol d'Amérique. 


\section{Quelques caractéristiques du parler jeune en espagnol}

En ce qui concerne la construction globale du discours, les études consacrées à cette variante linguistique signalent comme caractéristiques générales : a) la nette préférence des sujets parlants pour les procédés de coordination ou de simple juxtaposition, au lieu de se servir de la subordination ; b) l'introduction fréquente du discours d'autrui sans aucune marque de discours indirect, uniquement à l'aide d'un changement de la voix ; c) l'utilisation abondante d'éléments phatiques en vue de s'assurer de l'attention de l'interlocuteur ; d) une forte présence d'onomatopées, dans le but de rendre le récit plus vivant.

Du point de vue de la morphosyntaxe, les énoncés sont souvent construits sans prendre en compte l'accord sujet/verbe, et il est fréquent de trouver des mots ayant changé de catégorie grammaticale. Ainsi, cantidad, mazo et mogollón, appartenant à la catégorie nominale, sont souvent utilisés en tant qu'adverbes (pour les exemples, $c f$. plus loin les observations sur l'hyperbole).

Deux autres phénomènes fréquents : l'utilisation abondante de suffixes permettant de marquer 1'appartenance au groupe : -ata (bocata, fumata, drogata ...), -eta (fumeta, porreta...), -ota (drogota, pinchota...) et la troncation : anfetas (anfetaminas), pastis (pastillas), trapis (trapicheos). Est également présente, bien qu'à un moindre degré, l'agglutination suivie d'ellipse : finde (fin de semana), buenri (buen rollo).

Pour ce qui est du lexique, certains champs sémantiques sont fortement représentés, phénomène à mettre en rapport avec les observations de Gómez Capuz (2007), pour qui les centres d'intérêt des jeunes - notamment les jeunes marginaux - sont relativement limités, ce qui entraîne l'existence d'un nombre très abondant de métaphores synonymes renvoyant à une même notion (que l'auteur appelle pôle d'attraction synonymique). Cette surlexicalisation concerne très généralement le groupe d'appartenance, mais également des domaines tels que la musique, la drogue, l'argent ${ }^{(15)}$.

Pour désigner les amis et pour en parler, les jeunes de sexe masculin utilisent notamment los colegas, la gente, la peña, los chavales, la pandilla, la panda, los troncos, la basca, tandis que les filles préfèrent des formes plus clairement descriptives, telles que la gente con la que voy, el grupo con quien salgo.

Pour s'adresser aux membres du groupe, les garçons utilisent entre eux — de plus, très abondamment - colega, tío, tronco (tron), macho, chaval, etc. et pour les filles, beibi, piba, titi, mais aussi de nombreux termes qui, pris au sens littéral, constituent des insultes : cabrón, cabronazo, maricón, gilipollas ... Dans le même ordre d'idées, les échanges sont souvent ponctués de termes ayant à l'origine une valeur négative, mais qui sont devenus au fil du temps de simples éléments phatiques : coño, hostia, mierda, cojones...

Il faut noter également la présence abondante d'un lexique proche de l'hyperbole, que l'objectif de l'échange soit de caractériser un élément positif ou négatif. Ainsi, l'usage d'adjectifs ou d'adverbes intensificateurs est extrêmement fréquent : cojonudo, acojonante, de puta madre, mogollón, que te cagas, de la hostia, alucinante, flipante, a tope, de cojones, a lo bestia ou le plus poli súper, utilisé

(15) Pour une étude plus détaillée sur ces points, $c f$. notamment Betti (2006) et Mitkova (2007). 
comme préfixe pour des adjectifs (superinteresante, superenamorado...) ou des adverbes (superbien, supermal ...). Les expressions du type de ser una pasada, ser guay, ser la hostia sont également très courantes. Signalons enfin, l'utilisation de verbes indiquant « plaire» (molar, flipar, enrollar), accompagnés d'éléments emphatiques : mola mogollón, mola mazo, mola que te cagas... et l'utilisation abondante d'éléments intensificateurs au négatif : una chorrada, un puto $N$, un jodido $N$, un $N$ del carajo, un coñazo...

\section{En guise de conclusion}

Ce parcours comparatif nous a permis d'identifier plusieurs caractéristiques communes aux deux langues, à savoir : pour ce qui est des aspects morphosyntaxiques, le non respect de l'accord sujet/verbe, le changement de construction pour certains verbes ainsi que le changement de catégorie grammaticale pour certains noms et adjectifs, la transformation lexicale par troncation ou par suffixation ; pour les aspects purement sémantiques, un glissement de sens allant en général vers une restriction du sens par rapport au mot d'origine (en français, ce phénomène peut se produire après «verlanisation » du mot) ; pour les aspects pragmatiques, une présence très abondante de termes servant à s'adresser à l'interlocuteur ou à parler de ses pairs, ainsi que l'utilisation fréquente d'éléments purement phatiques. Nous insistons sur le fait qu'une partie importante de ces termes constituent des insultes en dehors de la situation énonciative considérée.

Bien que les caractéristiques mentionnées ne soient pas exclusivement réservées aux locuteurs jeunes, c'est bien chez eux que la présence de celles-ci est la plus abondante.

A travers ce parcours comparatif visant à illustrer le phénomène de détournement dans le langage des jeunes, nous espérons avoir mis clairement en avant le caractère créatif, dynamique et ludique de cette variété langagière, qui se sert en permanence de mots existants - dans la langue d'origine ou dans d'autres, via les emprunts - pour en créer d'autres. 


\section{Bibliographie}

Abecassis, M., Ayosso, L. et E. Vialleton (éds), (2007), Le français parlé au $X X I^{e}$ siècle : Normes et variations dans le discours en interaction, Paris, L'Harmattan (deux volumes).

ANSCOMBRE, J.-C. et S. MEJRI (éds), (2011), Le figement linguistique : la parole entravée, Paris, Honoré Champion.

ARMSTRONG, N. et M. JAMIN (2002), « Le français des banlieues : uniformity and discontinuity in the French of the Hexagon », K. Salhi (éds) French in and out of France : language policies, intercultural antagonisms and dialogue, Bern, Peter Lang, 107-136.

AUZANNEAU, M. et C. JUILlard (2012), « Jeunes et parlers jeunes : catégories et catégorisations », Langage et Société n 141, 5-20.

AuZAnNeAu, M., M. LeClÈRE-Messebel et C. JuILlard (2012), «Élaboration et théâtralisation de catégorisations linguistiques en discours, dans une séance de formation continue. La catégorie "jeune" en question », Langage et Société $\mathrm{n}^{\circ} 141,47-70$.

BARTA, P. (2005 et 2006), « Au pays des proverbes, les détournements sont rois », Paremia $\mathrm{n}^{\circ} 14,139-152$ (première partie) et Paremia $\mathrm{n}^{\circ} 15$, pp. 57-71 (deuxième partie).

BETTI, S. (2006), « La jerga juvenil de los SMS (-) » Cuadernos del Lazarillo ${ }^{\circ} 31$, Salamanca, 68-76.

CAUBET, D. (2008), « Des insultes aux vannes, ici et là-bas, en passant par les proverbes », Tauzin, A. (éds) Insultes, injures et vannes : en France et au Maghreb, Paris, Karthala, 111-134.

Cusimano, C. (2012), «Figement et séquences défigées », Texto! Textes et cultures [en ligne] Volume XVII n ${ }^{\circ} 14$.

Blanche-Benveniste, C. (2000), Approches de la langue parlée en français, Paris, Ophrys.

GADET, F. (2007), La variation sociale en français, Paris, Ophrys (nouvelle édition revue et augmentée).

Gómez CAPuZ, J. (2007), «El lenguaje del Neng de Castefa como estereotipo lingüístico de la subcultura dance y el argot juvenil actual », Revista Tonos Digital $\mathrm{n}^{\circ} 14$, Universidad de Murcia.

Gresillon, A. et D. Maingueneau (1984), « Polyphonie, proverbe et détournement, ou un proverbe peut en cacher un autre », Langages $n^{\circ} 73,112-125$.

GRÜNIG, B.-N. (1990), Les mots de la publicité, Paris, Presses du CNRS.

Kotschi, T., W. OSTERREICHER et K. ZIMMERMANN (éds) (1996), El español hablado y la cultura oral en España e Hispanoamérica, Vervuert Hispanoamericana.

LA GORGETTE, D. (2009), Les insultes en français : de la recherche fondamentale à ses applications (linguistique, littérature, histoire, droit), Editions de l'Université de Savoie.

LAKS, B. (1983), «Langage et pratiques sociales. Etude sociolinguistique d'un groupe d'adolescents ", Actes de la Recherche en Sciences Sociales $n^{\circ} 46,73$ 97.

LÉGLISE, I. et M. LEROY (2008), «Insultes et joutes verbales chez les “jeunes” : le re- 
gard des médiateurs urbains », A. Tauzin (éds), Insultes, injures et vannes : en France et au Maghreb, Paris, Karthala, 155-174.

LEXIK DES CITÉS ILLUSTRÉ, Paris, Editions Fleuve noir, 2007.

LUQUe, JdD., A. PAMIÉS, J. MANJÓN (1998), El arte del insulto : estudio lexicográfico, Barcelona, Península.

- (2000), Diccionario del insulto, Barcelona, Península.

Mitkova, A. (2007), «El léxico juvenil por áreas temáticas », Revista Tonos Digital $\mathrm{n}^{\circ} 14$, Universidad de Murcia.

PALMA, S. (2007), Les éléments figés de la langue. Etude comparative français-espagnol, Paris, L'Harmattan.

- (2008), «Le rôle des stéréotypes lexicaux dans les éléments figés de la langue », Des topoï à la théorie des stéréotypes en passant par la polyphonie te l'argumentation dans la langue (Hommages à Jean-Claude Anscombre), $\mathrm{Pu}-$ blications de l'Université de Savoie, 277-288.

- (2009), «Donde manda capital no manda guerrillero : estrategias enunciativas en los falsos refranes », Cuaderno de estudos linguisticos, 51 (1), Universidade de Campinas, São Paulo, 107-120.

RASTIER, F. (1997), "Défigements sémantiques en contexte », Martins-Baltar (éds.), La locution entre langue et usages, ENS Editions, 307-332.

SCHAPIRA, C. (1999), Les stéréotypes en français. Coll. L'essentiel français, Ed. Ophrys, Paris.

ZIMMERMANN, K. (1996), « Lenguaje juvenil, comunicación entre jóvenes y oralidad », Kotschi, T., W. Osterreicher et K. Zimmermann (éds), El español hablado y la cultura oral en España e Hispanoamérica, Vervuert Hispanoamericana, 475-514.

Zuluaga, A. (1999), « Traductología y fraseología », Paremia n8, 537-549. 